\title{
Characterization of Solidified Gas Thin Film Targets via Alpha Particle Energy Loss
}

\author{
M.C. Fujiwara ${ }^{\mathrm{a}, 1}$, G.A. Beer ${ }^{\mathrm{b}}$, J.L. Beveridge ${ }^{\mathrm{c}}$, J.L. Douglas ${ }^{\mathrm{b}}$, \\ T.M. Huber ${ }^{\mathrm{d}}$, R. Jacot-Guillarmod ${ }^{\mathrm{e}}$, S.K. Kim ${ }^{\mathrm{f}}$, \\ P.E. Knowles ${ }^{\text {b, }, ~ A . R . ~ K u n s e l m a n ~}{ }^{g}$, M. Maier ${ }^{\text {b }}$, \\ G.M. Marshall ${ }^{\mathrm{c}}$, G.R. Mason ${ }^{\mathrm{b}}$, F. Mulhauser ${ }^{\mathrm{c}, 3}$, A. Olin ${ }^{\mathrm{c}}$, \\ C. Petitjean ${ }^{\text {h}}$, T.A. Porcelli ${ }^{\mathrm{b}}$ and J. Zmeskal ${ }^{\mathrm{i}}$ \\ Muonic Hydrogen Collaboration \\ ${ }^{a}$ Department of Physics and Astronomy, University of British Columbia, \\ Vancouver, BC, V6T 2A6 Canada \\ ${ }^{\mathrm{b}}$ Department of Physics and Astronomy, University of Victoria, Victoria, BC, \\ V8W 2 Y2 Canada \\ ${ }^{\mathrm{c}}$ TRIUMF, Vancouver, BC, V6T $2 A 3$ Canada \\ ${ }^{\mathrm{d}}$ Department of Physics, Gustavus Adolphus College, St. Peter, MN 56082, USA \\ ${ }^{\mathrm{e}}$ Physics Institute, Université de Fribourg, CH-1700 Fribourg, Switzerland \\ ${ }^{\mathrm{f}}$ Department of Physics, Jeonbuk National University, \\ Jeonju City 560-756, S. Korea \\ ${ }^{\mathrm{g}}$ Department of Physics, University of Wyoming, Laramie, WY 82071, USA \\ h Paul Scherrer Institute, CH-5232 Villigen, Switzerland \\ ${ }^{\mathrm{i}}$ Austrian Academy of Sciences, A-1090 Wien, Austria
}

\begin{abstract}
A method is reported for measuring the thickness and uniformity of thin films of solidified gas targets. The energy of $\alpha$ particles traversing the film is measured and the energy loss is converted to thickness using the stopping power. The uniformity is determined by measuring the thickness at different positions with an array of sources. Monte Carlo simulations have been performed to study the film deposition mechanism. Thickness calibrations for a TRIUMF solid hydrogen target system are presented.
\end{abstract}




\section{Introduction}

Recently, the use of thin films of solidified gases has attracted attention in particle beam experiments [1-21]. A novel target system has been developed at TRIUMF for making films of solid hydrogen isotopes and other gases for experiments in muon catalyzed fusion [1-3]. Several measurements have been reported using the apparatus [4-12] and more are proposed for the future [1317]. Similar layers of solid hydrogen isotopes have been utilized in an attempt to produce a source of low energy negative muons [18-20]. Production of a muonic helium ion beam of keV energies [21] as well as MeV energies [13,14] has been proposed using multilayer cryogenic films.

In these experiments, characterization of the target films is often important in the analysis of the experimental data. For cross section measurements, in particular, the accurate knowledge of the thickness and uniformity of the target films is essential, since the uncertainty in the thickness directly propagates to the final results.

With the TRIUMF target system, solid layers of hydrogen isotopes (protium ${ }^{1} \mathrm{H}_{2}$, deuterium, tritium and their mixtures) and other gases such as neon can be made with thicknesses ranging from a few $\mu \mathrm{g} \cdot \mathrm{cm}^{-2}(\sim 1 \mu \mathrm{m}$ for protium) to a few $\mathrm{mg} \cdot \mathrm{cm}^{-2}$. Due to the spatial and cryogenic limitations, conventional methods for thin film thickness measurement, such as optical interferometry, cannot be used with our system.

Thickness measurements of condensed gases have been reported by several authors. Sørensen et al. used a quartz crystal oscillator to measure solid hydrogen film thicknesses [22]. However, the method suffered from a severe non-linearity, which was attributed to the low density of hydrogen. Rutherford Backscattering (RBS) [23] which was used, for example, by Chu et al. [24] to measure the thickness of solid argon, oxygen, and $\mathrm{CO}_{2}$, is kinematically impossible for protium targets.

In the present work, we have used the energy loss of $\alpha$ particles traversing the film to measure the thickness. Uniformity was determined by measuring the thickness at different positions with an array of sources. Following the introduction, details of the experimental apparatus are described in section 2. Sections 3 discusses data analysis and uncertainties. The results of various

\footnotetext{
$\overline{1}$ Corresponding author, Tel: +1 6042221047 (ext. 6290), Fax: +1 604222 1074, e-mail: fujiwara@triumf.ca

2 Present address: Université Catholique de Louvain, B-1348 Louvain-La-Neuve, Belgium

3 Present address: Physics Institute, Université de Fribourg, CH-1700 Fribourg, Switzerland
} 
measurements are presented in section 4, and comparison with Monte Carlo simulations, as well as discussion is given in section 5 .

\section{Experimental apparatus}

Figure 1 shows a schematic view of the experimental apparatus. The general description of the target system including gas deposition mechanism is given in Refs. [1,2]. A linear array of five alpha spot source was custom-manufactured 4 by electro-deposition of ${ }^{241} \mathrm{Am}$ onto a gold-plated oxygen-free copper plate. The spot sources had nominal diameters of $3 \mathrm{~mm}$, a center-to-center separation of $10 \mathrm{~mm}$, and were covered by a thin gold layer $\left(\sim 200 \mu \mathrm{g} \cdot \mathrm{cm}^{-2}\right)$ for safety and ease of handling. The plate, enclosed in an evacuated chamber, was cooled to approximately $3 \mathrm{~K}$, and hydrogen gas solidified onto it when introduced through the gas diffusing mechanism (diffuser). Different gas deposition systems have been developed to incorporate experimental demands, and to improve the performance $[1,2]$. For system 1, the gas was released through a diffuser made of a thin stainless steel foil perforated with many holes $(\sim 0.2$ mm diameter). System 2 used the same diffuser foil, but as a result of gas line modifications to incorporate tritium compatibility, it had gas inlet tubing with a higher volume, which acted as an unwanted buffer volume as we shall see in section 4.1. System 3 is the latest version with the gas inlet lines replaced with low volume, high conductance tubing, and a sintered metal diffuser (2 $\mu \mathrm{m}$ porosity) employed to ensure microscopic homogeneity of deposition.

The distance between the diffuser surface and the target plate, which is adjustable depending on the experimental requirements, was for the present measurements about $14 \mathrm{~mm}$ for System 1 and 2, and about $8 \mathrm{~mm}$ for System 3.

The amount of gas injected into the system was measured in units of Torr-litre (abbreviated $\mathrm{T} \cdot l$ ), where one $\mathrm{T} \cdot l$ corresponds to the number of molecules in one litre of gas at a pressure of 1 Torr and ambient temperature $(\sim 295 \mathrm{~K})$. This unit was operationally convenient since the number of molecules can be compared, independent of the isotopic composition.

The rate of gas deposition was typically of the order of a few $(\mathrm{T} \cdot l) \cdot s^{-1}$ or less, the limit imposed by a requirement to avoid significant intermolecular interactions which would lead to heat conduction from the relatively hot $(\sim 100$ $\mathrm{K})$ diffuser to the cold plate $(\sim 3 \mathrm{~K})$.

Alpha particles penetrating the hydrogen film were detected by a passivated,

$\overline{4}$ Isotope Products Laboratories, 1800 N. Keystone St., Burbank, CA, USA. 
implanted planar silicon detector 5 of active thickness $150 \mu \mathrm{m}$ and area 600 $\mathrm{mm}^{2}$. The detector was mounted on the diffuser frame which was part of a mechanism that allowed the diffuser to be inserted and retracted (Fig. 1). The detector thus moved vertically to allow a measurement of the thickness at different positions by detecting the $\alpha$ particles from each of the five spot sources. A collimating device which consisted of an array of small holes (diameter $\sim 1 \mathrm{~mm}$ ) restricted the angular path of the $\alpha$ particles to accept alphas from only one spot source at a time. The signal from the detector was recorded with a standard spectroscopy system consisting of a charge sensitive preamplifier, linear amplifier, and analog-to-digital converter, together with a CAMAC/VAXstation data acquisition system. The energy scale of the alpha detection system was calibrated using a separate ${ }^{241} \mathrm{Am}$ source. Calibration was frequently required since temperature variations in the detector could cause significant shifts in the gain. The system achieved a typical resolution of $0.4 \%$ (FWHM) at a detector temperature near $100 \mathrm{~K}$. The profile of alpha counts versus the vertical position of the collimated detector for a bare target (i.e. no solid hydrogen layer) is shown in Fig. 2. The plot confirms that we detected $\alpha$ particles from only one source spot at a time.

\section{Analysis}

\subsection{Thickness determination}

Figure 3 shows an example of the energy spectra of $\alpha$ particles penetrating hydrogen films with different amounts of gas injected, namely 0, 150, and $300 \mathrm{~T} \cdot l$. The shift of the peaks to lower energy with increased injected gas is clearly visible. Note also the peak broadening (due mainly to straggling) and the asymmetric peak shape which is due in part to the energy loss in the protective gold layer on the source. We determine the mean energy value $\langle E\rangle$ from the centroid of the energy distribution $f(E)$ in the spectrum via

$$
<E>=\frac{\int_{<E>-\epsilon}^{<E>+\epsilon} f(E) E d E}{\int_{<E>-\epsilon}^{<E>+\epsilon} f(E) d E},
$$

where $\epsilon$ is a finite cutoff value. The use of the centroid in the analysis achieves sufficient accuracy while avoiding the difficulties in fitting the irregular peak

5 Canberra, model FD/S-600-29-150-RM. 
shapes, which vary depending on the source spot and target thickness.

In the approximation that the angular dispersion of $\alpha$ particles is avoided due to the use of a collimator, the thickness of the target $T$ can be obtained from $R(<E>)$, the alpha range as a function of energy:

$$
T=R\left(<E_{\text {init }}>\right)-R\left(<E_{\text {fin }}>\right),
$$

where $\left\langle E_{\text {init }}\right\rangle$ is the initial energy of the $\alpha$ particles and $\left\langle E_{\text {fin }}\right\rangle$, the energy after traversing the target.

For our analysis, the stopping power or the range in the solid state of hydrogen for $\alpha$ particles in the energy range of $\sim 2-5 \mathrm{MeV}$ was needed, but no experimental data is available for solid hydrogen at these energies. The detailed discussion of the effect of physical phase on stopping power for heavy charged particles, which is reported for keV projectiles in hydrogen [25] and in nitrogen [26], as well as for $\mathrm{MeV}$ ions in organic and other materials [27-29], can be found in Ref. [13] (see also reviews [30-32]). After a critical survey of the literature [13], a recent compilation for gaseous hydrogen by the International Commission of Radiation Units and Measurements (ICRU) [33] was used in the analysis.

\subsection{Uncertainties}

The systematic uncertainties considered in the analysis include knowledge of stopping power, the effect of energy cuts, and energy calibration of the detector. The uncertainty in the stopping power is difficult to estimate, since neither experimental data nor satisfactory theory is available for our case. The ICRU table [33] claims an accuracy of $\sim 1-2 \%$ at $4 \mathrm{MeV}$ and $\sim 2-5 \%$ at $1 \mathrm{MeV}$ for gaseous hydrogen.

Ziegler et al., in another commonly used compilation [34], give an estimate of stopping power of solids for which there are no data available, by interpolating (or, for hydrogen, extrapolating) the data from other elements under certain assumptions. They quote a $5 \%$ average accuracy for $\alpha$ particle stopping powers in solids, which is simply the average of deviations taken from the collections of experimental data in the literature for many elements.

The two tables ([33] and [34]) agree with each other within $3 \%$ at $\sim 2 \mathrm{MeV}$, and the thicknesses derived using both tables agree within $2 \%$ (the differences in stopping powers partly cancel with one another upon integration over energy). We shall conservatively quote $5 \%$ of the derived thickness as the uncertainty due to the stopping power, including a possible physical phase effect. This was 
the limit on our accuracy in most cases.

The effect of the finite cutoff values ( $\epsilon$ in Eq. 1) in determining the centroid of the energy spectra was investigated by changing the cuts and its uncertainty was found to be small compared to that from the stopping powers in most cases. The uncertainty in the detector energy calibration is estimated to be less than $2 \mathrm{keV}$, except for a few measurements during which a temperature variation in the silicon detector resulted in a gain shift.

These systematic uncertainties, as well as the statistical uncertainties, were added quadratically to obtain the total uncertainty. Except for very thin layers, whose thicknesses have to be determined from a small difference in the initial and final alpha energies, the knowledge of the stopping power dominated the uncertainty.

\section{Results}

\subsection{Linearity of film deposition}

The linearity of the deposition was tested by measuring the thickness of films made with different amounts of gas input under different conditions. The data shown in Fig. 4 were taken at the central source position $(0 \mathrm{~mm})$ for films made by diffuser system 1 . Some of the films $(40,300 \mathrm{~T} \cdot l)$ were made by depositing gas on top of existing films, whereas others $(20,150,400 \mathrm{~T} \cdot l)$ were made with a single deposition. The solid line represents a weighted least-squares fit to the data (plotted with error bars ${ }^{\emptyset}$ ). The slope represents the conversion factor from gas input to the film thickness at this source position $(0 \mathrm{~mm})$. A possible non-linear component in deposition was investigated by allowing a quadratic term in the fitting function. The maximum allowable non-linear contribution, when extrapolated to a $1000 \mathrm{~T} \cdot l$ target, was similar in size to the uncertainty in the thickness due to the stopping power. Similarly, the data from other source positions on the plate, and with diffuser systems 2 and 3, showed good linearity for these moderate thicknesses.

The extrapolation of the results to very thin films requires some caution; if a small amount of the gas remained in the gas transfer tubes, it would be lost from the layer, giving a small offset in gas deposition. This gas loss is negligible for thick layers, but can be important for thin layers. The effect was examined for systems 2 and 3 by comparing two series of measurements; (1)

$\overline{6}$ Error bars presented in this paper represent total uncertainties including that from stopping power, unless otherwise stated. 
thick films made by a single deposition of a large amount of input gas, in which the gas loss is negligible, and (2) thin films made by sequential depositions of small amounts of gas, where the gas loss from each deposition, if it exists, is multiplied to give a measurable effect after several depositions.

Due to the small energy loss, measurement of very thin films was difficult, and we have assumed that the same cutoff value, $\epsilon$ in Eq. 1, can be used to determine both the initial and the final energies (this is not generally valid in the thick film measurement due to the peak broadening). Provided that the same value is used for both initial and final spectra, the choice of $\epsilon$ did not affect the resulting thickness values.

Figures 5(a) and 5(b) show the results of the comparisons for systems 2 and 3 respectively. For system 2 (Fig. 5(a)), sequential deposition of small amounts of gas (measurement (2), filled squares) resulted in a smaller thickness per unit gas input than thick films made with a single deposition (measurement (1), open squares), indicating that when making very thin films a non-negligible amount of the gas remains in the gas transfer tubes without being deposited. On the other hand, System 3 (Fig. 5(b)), which was in fact designed to remove the effect, shows no evidence of such gas loss.

\subsection{Thickness profile}

Shown in Fig. 6 are the thickness profiles from different deposition systems. An asymmetric non-uniformity with respect to the center can be observed. The error bars do not include the uncertainty from the stopping power, since it is not relevant when considering relative uniformity.

One may assume that the thickness of a film at a particular position depends only on the relative distance from the diffuser, which is the case if, for example, the molecules stick onto the cold plate at the first contact as suggested from the measurements described in Section 4.3. In this model, the film profile should have translational invariance under the diffuser displacement.

A series of measurements were made using system 3 with films that were deposited with the diffuser displaced by $0 \mathrm{~mm},+5 \mathrm{~mm},+10 \mathrm{~mm}$ and -20 $\mathrm{mm}$ from the nominal standard position, the positive direction being upward. In Fig. 7, the resulting thicknesses are plotted against diffuser coordinates, i.e., the relative vertical distance from the center of the diffuser, unlike Fig. 6 which was plotted against the distance from the center of the source plate. The data from different films, thus plotted, are consistent, indicating the validity of translation invariance of the film profile. One exception is the filled star point at distance $0 \mathrm{~mm}$ in the diffuser coordinates, which represents the thickness at the center of the diffuser for a film deposited with the diffuser retracted 20 
$\mathrm{mm}$ downwards. At this position the diffuser, which has an active diameter of $60 \mathrm{~mm}$, was aligned with the bottom source spot (see Fig. 1) and a significant fraction of emitted gas molecules missed the cold plate, hence the molecules may bounce around inside the vacuum system, eventually sticking nearer to the bottom rather than the top of the cold plate. Nevertheless, validity of the translational invariance indicated above suggests that it is possible to measure a thickness at an arbitrary point along the vertical axis with the present technique, despite the fact that $\alpha$ particles are emitted only from discrete source spots. Figure 7 can thus be considered as a good representation of the thickness profile of the film made with system 3. Based on these results, the calibration factors (thickness per unit gas input) for system 3 at each position with respect to the diffuser center, including the $5 \%$ uncertainty from the stopping power, are given in Table 1 . When more than one measurement exist for the same position, an average was taken, except that some points from the diffuser position of $-20 \mathrm{~mm}$, which may be in error as discussed, were not included in the final average.

It should be noted that, in general, the calibration factor depends on the distance between the diffuser surface and the cold plate surface which, in the present case, was $8 \mathrm{~mm}$. For system 1, which had a $14 \mathrm{~mm}$ separation between the diffuser and cold plate, the calibration factors at the standard vertical diffuser position are given in Table 2. The values for system 2 are similar and agree with system 1 within the uncertainty (see Fig. 6).

Thicknesses of deuterium and tritium in units of $\mu \mathrm{g} \cdot \mathrm{cm}^{-2}$ are factors of 2 and 3, respectively, larger than for a protium film with the same number of molecules due to the isotopic mass difference. The measurement of a deuterium film, when corrected by this factor, showed good agreement with protium films.

\subsection{Other measurements}

Measurements were made with films deposited under different conditions to see the effects on thickness and uniformity. No deviation was found for films made with and without pumping the target vacuum during deposition within the relative uncertainty of about $1 \%$. Reducing the deposition rate by an order of magnitude also did not noticeably affect either thickness or uniformity.

The gas deposition system is capable of making a second film on a separate cold surface through the opposite side of the diffuser [2]. The apparatus was designed to minimize unwanted cross deposition from one side to the other by shielding with cold surfaces. By intentionally releasing a large amount of gas through the opposite side, cross deposition on the spot source target was checked. The measurement, made with similar assumptions on the cutoff value 
$\epsilon$ to the measurement of very thin films described in Section 4.1, indicated that less than one part in a thousand of the injected gas arrived at the central spot. This result, together with the fact that pumping the system did not affect deposition, suggests that the gas molecules are likely to stick to the cold surface on first contact, which would explain the observed translational invariance of film profiles (Section 4.2).

Beam experiments using solid hydrogen targets sometimes last for a few days, so it is important to check the effect of target aging. The results of measurements, made 8 hours apart, of the same film were consistent with each other, giving an upper limit in thickness variation $\Delta T / T \leq 0.5 \%$ over 8 hours. In the analysis, a similar assumption in the cutoff value was made.

Films of other gases can also be deposited with the target system (for example, neon films have been used in TRIUMF experiments [6,11]). Thickness measurements of neon films were made using two different gas inputs at three of the five source positions. The stopping power in gaseous neon taken from the ICRU tables [33] was used to convert energy loss to thickness. The same $5 \%$ uncertainty in stopping power was assumed. Calibration factors for the three spots are given in Table 3 .

Another method of deposition was tested using system 1; the diffuser was completely moved away from the cold target plate, and a relatively large amount of gas $(300 \mathrm{~T} \cdot l)$ was introduced into the system with all the pump valves closed. The vacuum pressure during deposition was kept relatively high to allow some interactions among the molecules. The thickness profile of the film is shown in Fig. 8. Notice the much lower deposition efficiency compared to normal film deposition shown in Fig. 6. The uncertainties are relatively large due to the small thickness and a noise problem experienced during some of the measurements. Nevertheless, the results suggest a more uniform film deposition, so this approach may be useful in certain cases in which uniformity is critical.

\section{Discussion}

\subsection{Monte Carlo simulation}

In order to better understand the mechanism of gas deposition, Monte Carlo simulations were performed with the following assumptions: (1) molecules are emitted uniformly from the gas diffuser surface, (2) the molecules stick to the cold surface at the position of first contact, and (3) there is no interaction between the molecules. The last assumption is justified since the requirement to

keep the target film temperature cold demands an insignificant intermolecular 
interaction, minimizing heat conduction from the warmer diffuser to the target plate. Pressure must be maintained low enough during deposition to keep the mean free path of the emitted molecules comparable to or larger than the diffuser-to-plate separation. Three different models for the angular distribution of molecules emitted from the diffuser surface were used in simulations; (a) the (unrealistic) forward emission model assuming $\theta=0$, where $\theta$ is the angle with respect to the normal to the diffuser surface, (b) the isotropic emission model assuming that the number of molecules emitted into unit solid angle $d w$ is constant, independent of $\theta$, i.e. $d w / d(\cos \theta)=$ const., and (c) the $\cos \theta$ emission model using a diffusion-like angular distribution $d w / d(\cos \theta) \propto \cos \theta$. The diffuser diameter of $60 \mathrm{~mm}$ and the distance of $8 \mathrm{~mm}$ between the diffuser and cold plate surface were used in simulations to compare with the measurements for system 3. There are no free parameters in the simulations other than the emission angular distribution. Figure 9 compares the simulation results with the film thickness profile for system 3 as deduced above, which is plotted with error bars that now include the uncertainty from stopping power. The agreement with the $\cos \theta$ model (c) is quite good, except for the asymmetry in the shape.

The asymmetry, which obviously cannot be reproduced with our simulations, is unlikely to be due to an effect of gravity on the target, since the relative non-uniformity remains constant over a wide range of the thickness. The shape may be partly explained by the fact that the gas is introduced from the bottom of the diffuser system, hence gas molecules may have a larger probability of diffusing out at the bottom rather than the top of the diffuser, indicating a breakdown of assumption (1).

Similar simulations with the diffuser-to-plate distance taken to be $14 \mathrm{~mm}$ were compared to data for system 1 . The $\cos \theta$ model best describes the data, but comparison indicates a slightly more forward peaked emission angular distribution. It should be recalled, however, that system 1 had a different perforation structure from system 3 .

Reasonable success in the simulation with the $\cos \theta$ model gives us some confidence in scaling the thickness as a function of distance between the diffuser surface and the target support foil surface. Figure 10 shows a comparison of the simulated thickness profiles with different distances.

\subsection{Effective thickness}

The results given in Tables $1-3$ should be used with caution when the films are used as a target for beam experiments. Since the present work measures the profile only in the vertical dimension, the profile in the horizontal dimension 
has to be assumed when estimating an average target thickness. For a nonuniform layer, the average thickness depends on the width and profile of the beam which stops in the target. The angular divergence of the beam also contributes to the effective thickness.

An effective average thickness can be defined via

$$
T^{e f f}=\frac{\sum w_{i} T_{i}}{\sum w_{i}}
$$

where $T_{i}$ is the thickness at the $i$ th measured spot, and $w_{i}$ the weighting factor. A weighted root-mean-square deviation of thickness is defined via:

$$
\Delta T_{r m s}^{e f f}=\sqrt{\frac{\sum w_{i}\left(T_{i}-T^{e f f}\right)^{2}}{\sum w_{i}}} .
$$

This is a quantitative measure of the non-uniformity and is useful when optimizing the vertical position of the diffuser for deposition.

The weighting factors depend on the beam parameters and the type of measurement. For example, when the target is used to stop particle beams from an accelerator as in Ref. [4], a gaussian distribution with a certain width may be justified. However, when muonic hydrogen atoms emitted from a solid hydrogen layer are stopped in a deuterium film, as in Ref. [8], the atomic beam is divergent with an angular distribution close to $d w / d(\cos \theta) \propto \cos \theta$, and depends on scattering cross sections. In this case the detailed averaging has to be done with a Monte Carlo simulation which includes differential scattering cross sections.

\section{Conclusions}

Deposited films of solid hydrogen isotopes and neon have been characterized via the energy loss of $\alpha$ particles. The present method can be applied to a relatively wide range of film thicknesses, e.g. for protium, from $\sim 1 \mu \mathrm{g} \cdot \mathrm{cm}^{-2}$ to $\sim 1 \mathrm{mg} \cdot \mathrm{cm}^{-2}$. The accuracy of the measurement is limited by the uncertainty in the stopping powers, but the relative accuracy can reach better than $1 \%$. The uniformity can be measured by sampling the thickness at different positions with an array of alpha sources. Furthermore, it was possible to determine the thickness at an arbitrary vertical position by measuring films deposited with different diffuser positions. We note that muon catalyzed fusion reactions could also be used as a mono-energetic alpha source for the thickness measurement [13] (see also [20]). 
For the TRIUMF target system, the linearity of deposition was confirmed, with the exception of very thin film deposition with system 2 . An asymmetric non-uniformity was observed in all films, while deposition conditions (such as pumping of the high vacuum region surrounding the target, or change in gas deposition rate) did not affect the thickness or uniformity. Deposition from one side of the diffuser did not contaminate the cold plate on the other side to a limit of one part in a thousand, enabling experiments using two separated solid hydrogen targets [5]. No evidence was seen for the change in the film thickness over time. The comparison with Monte Carlo simulations indicates that the angular distribution of gas emission is close to a $\cos \theta$ model. The thickness calibration as well as film profile for the TRIUMF target is presented (Tables $1-3)$.

\section{Acknowledgement}

The authors thank Professors D.F. Measday, W.N. Hardy and M. Senba for their valuable suggestions and discussions. We gratefully acknowledge support of the Natural Sciences and Engineering Research Council of Canada, and the Swiss National Science Foundation. M.C.F. thanks Rotary Foundation, the University of British Columbia and the Department of Foreign Affairs and International Trade of the Government of Canada for their support.

\section{References}

[1] P. E. Knowles et al., Hyperfine Int. 82, 521 (1993).

[2] P. E. Knowles et al., Nucl. Instr. Meth. A 368, 604 (1996).

[3] M. C. Fujiwara et al., Hyperfine Int. 101/102 (1996), in press. [TRIUMF preprint: TRI-PP-95-91]

[4] B. M. Forster et al., Hyperfine Int. 65, 1007 (1990).

[5] G. M. Marshall et al., Hyperfine Int. 82, 529 (1993).

[6] R. Jacot-Guillarmod et al., Hyperfine Int. 82, 501 (1993).

[7] F. Mulhauser et al., in Muons and Pions in Matter, ed. by JINR, p227 (1995).

[8] F. Mulhauser et al., Phys. Rev. A 533069 (1996).

[9] G. M. Marshall et al., Hyperfine Int. 101/102 (1996), in press. [TRIUMF preprint: TRI-PP-95-89]

[10] P. E. Knowles et al., Hyperfine Int. 101/102 (1996), in press. [TRIUMF preprint: TRI-PP-95-92] 
[11] R. Jacot-Guillarmod et al., Hyperfine Int. 101/102 (1996), in press.

[12] M. C. Fujiwara et al., Proceedings of International Conference on Muon Spin Rotation/Relaxation/Resonance, Nikko, Japan, 1996, to be published in Hyperfine Int. [TRIUMF preprint: TRI-PP-96-45]

[13] M. C. Fujiwara, Master's thesis, University of British Columbia, unpublished (1994).

[14] M. C. Fujiwara et al., Hyperfine Int. 101/102 (1996), in press. [TRIUMF preprint: TRI-PP-95-90]

[15] M. C. Fujiwara et al., TRIUMF Research Proposal E767, 1995.

[16] R. Jacot-Guillarmod et al., Hyperfine Int. 101/102 (1996), in press.

[17] V.M. Bystritsky et al., TRIUMF Research Proposal E742, 1994.

[18] K. Nagamine, Proc. Japan Academy 65, 225 (1989).

[19] P. Strasser et al., Hyperfine Int. 101/102 (1996), in press.

[20] P. Strasser et al., Phys. Lett. B. 368, 32 (1996).

[21] K. Nagamine et al., Hyperfine Int. 82, 343 (1993).

[22] H. Sørensen, Appl. Phys. 9, 321 (1976).

[23] See, for example W. K. Chu et al., Backscattering Spectrometry (Academic Press, Inc., New York, New York, 1978).

[24] W. K. Chu et al., Nucl. Instr. and Meth. 149, 115 (1978).

[25] P. Børgesen, Nucl. Instr. and Meth. 200, 571 (1982).

[26] P. Børgesen, Nucl. Instr. and Meth. 194, 71 (1982).

[27] J. F. Ziegler and W. K. Chu, J. Appl. Phys. 47, 2239 (1976).

[28] D. I. Thwaites and D. E. Watt, Phys. Med. Biol. 23, 426 (1978).

[29] D. I. Thwaites, Radiat. Res. 95, 495 (1983).

[30] P. Børgesen, Nucl. Instr. and Meth. B 12, 73 (1985).

[31] D. I. Thwaites, Nucl. Instr. and Meth. B 12, 84 (1985).

[32] D. I. Thwaites, Nucl. Instr. and Meth. B 27, 293 (1987).

[33] ICRU Report 49, Stopping Powers and Ranges for Protons and Alpha Particles (International Commission of Radiation Units and Measurements, Bethesda, Maryland, 1993).

[34] J. F. Ziegler et al., The Stopping and Range of Ions in Solids (Pergamon Press, New York, 1985). 
Table 1

Protium film thicknesses at various distance from the center of the film for system 3. The distance between the diffuser surface and cold plate surface was $8 \mathrm{~mm}$.

\begin{tabular}{c|c}
\hline $\begin{array}{c}\text { Position with respect to } \\
\text { film center }(\mathrm{mm})\end{array}$ & $\begin{array}{c}\text { Thickness per unit gas input } \\
\left(\mu \mathrm{g} \cdot \mathrm{cm}^{-2} \cdot(\mathrm{T} \cdot \mathrm{l})^{-1}\right)\end{array}$ \\
\hline 40 & $0.21 \pm 0.08$ \\
30 & $1.52 \pm 0.11$ \\
20 & $2.92 \pm 0.17$ \\
15 & $3.10 \pm 0.18$ \\
10 & $3.21 \pm 0.16$ \\
5 & $3.33 \pm 0.19$ \\
0 & $3.46 \pm 0.17$ \\
-5 & $3.64 \pm 0.18$ \\
-10 & $3.76 \pm 0.19$ \\
-15 & $3.92 \pm 0.21$ \\
-20 & $3.77 \pm 0.19$ \\
-25 & $3.06 \pm 0.17$ \\
-30 & $1.68 \pm 0.09$ \\
\hline
\end{tabular}


Table 2

Thickness of the protium film for system 1, deposited with standard diffuser position.

\begin{tabular}{c|c}
\hline $\begin{array}{c}\text { Source position } \\
(\mathrm{mm})\end{array}$ & $\begin{array}{c}\text { Thickness per unit gas input } \\
\left(\mu \mathrm{g} \cdot \mathrm{cm}^{-2} \cdot(\mathrm{T} \cdot \mathrm{l})^{-1}\right)\end{array}$ \\
\hline 20 & $2.37 \pm 0.13$ \\
10 & $2.94 \pm 0.15$ \\
0 & $3.32 \pm 0.17$ \\
-10 & $3.60 \pm 0.19$ \\
-20 & $3.31 \pm 0.17$ \\
\hline
\end{tabular}

Table 3

Thickness calibration factors for a neon film at source positions $-20 \mathrm{~mm}, 0 \mathrm{~mm}$ and $20 \mathrm{~mm}$.

\begin{tabular}{c|c}
\hline $\begin{array}{c}\text { Source position } \\
(\mathrm{mm})\end{array}$ & $\begin{array}{c}\text { Thickness per unit gas input } \\
\left(\mu \mathrm{g} \cdot \mathrm{cm}^{-2} \cdot(\mathrm{T} \cdot l)^{-1}\right)\end{array}$ \\
\hline 20 & $24.7 \pm 1.7$ \\
0 & $32.5 \pm 2.0$ \\
-20 & $31.5 \pm 1.9$ \\
\hline
\end{tabular}




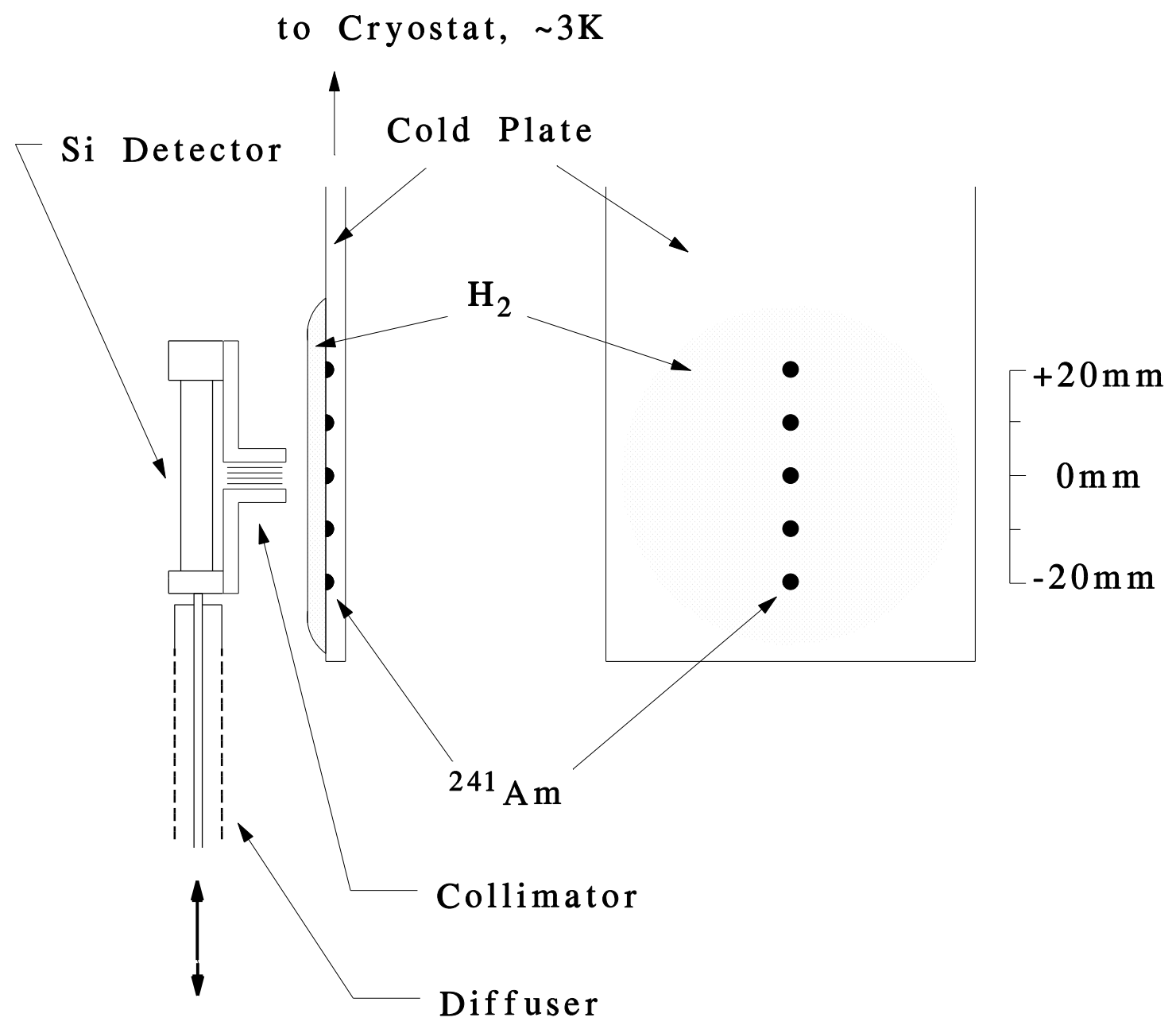

\section{Side View Front View}

Fig. 1. Schematic view of the experimental setup with solid hydrogen $\left(\mathrm{H}_{2}\right)$ layer.

The apparatus is contained in an evacuated chamber. 


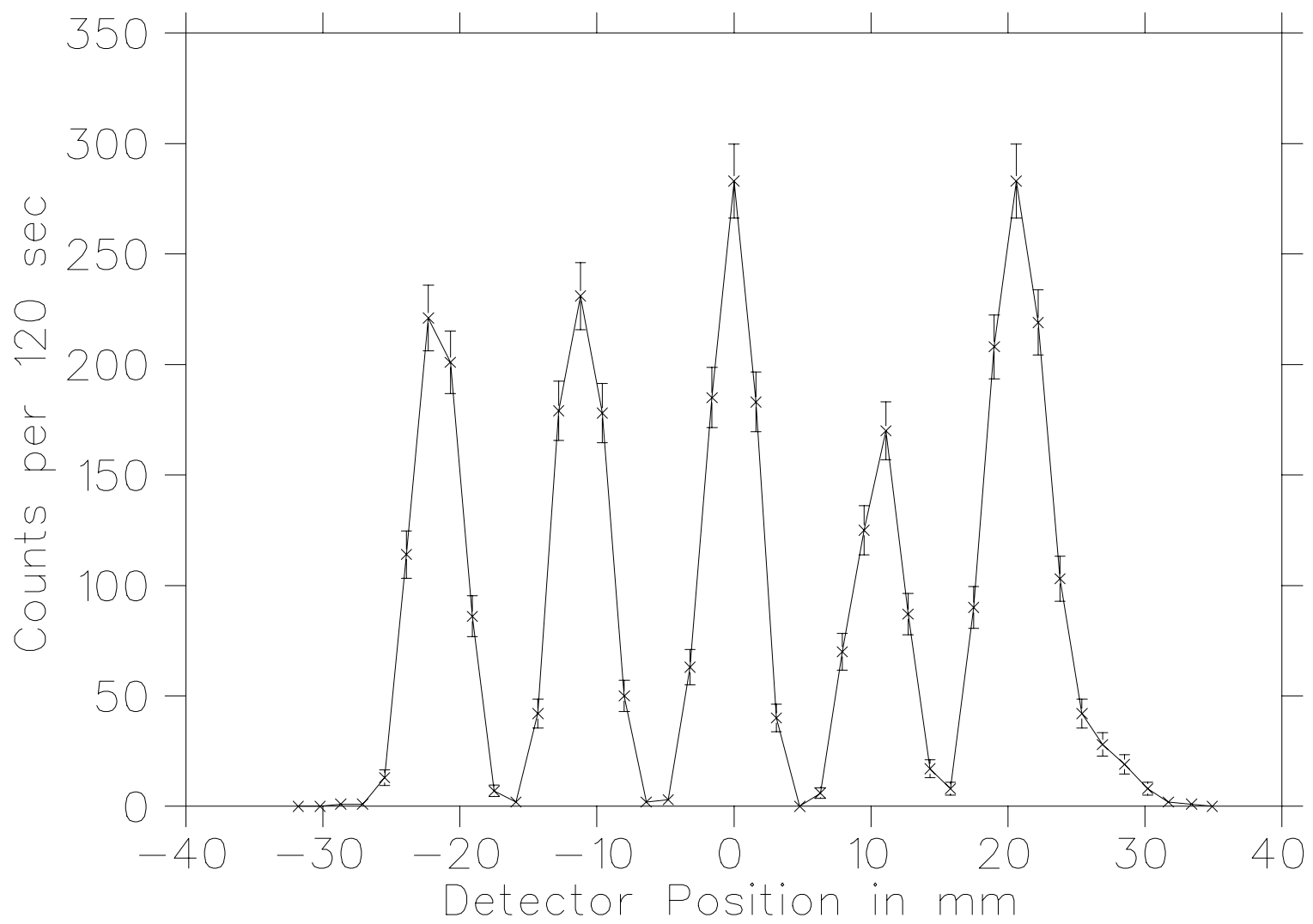

Fig. 2. Counts versus vertical position of the silicon detector with respect to the center. Each peak corresponds to one of the five source spots. The detector is collimated such that it sees only one spot at a time. 


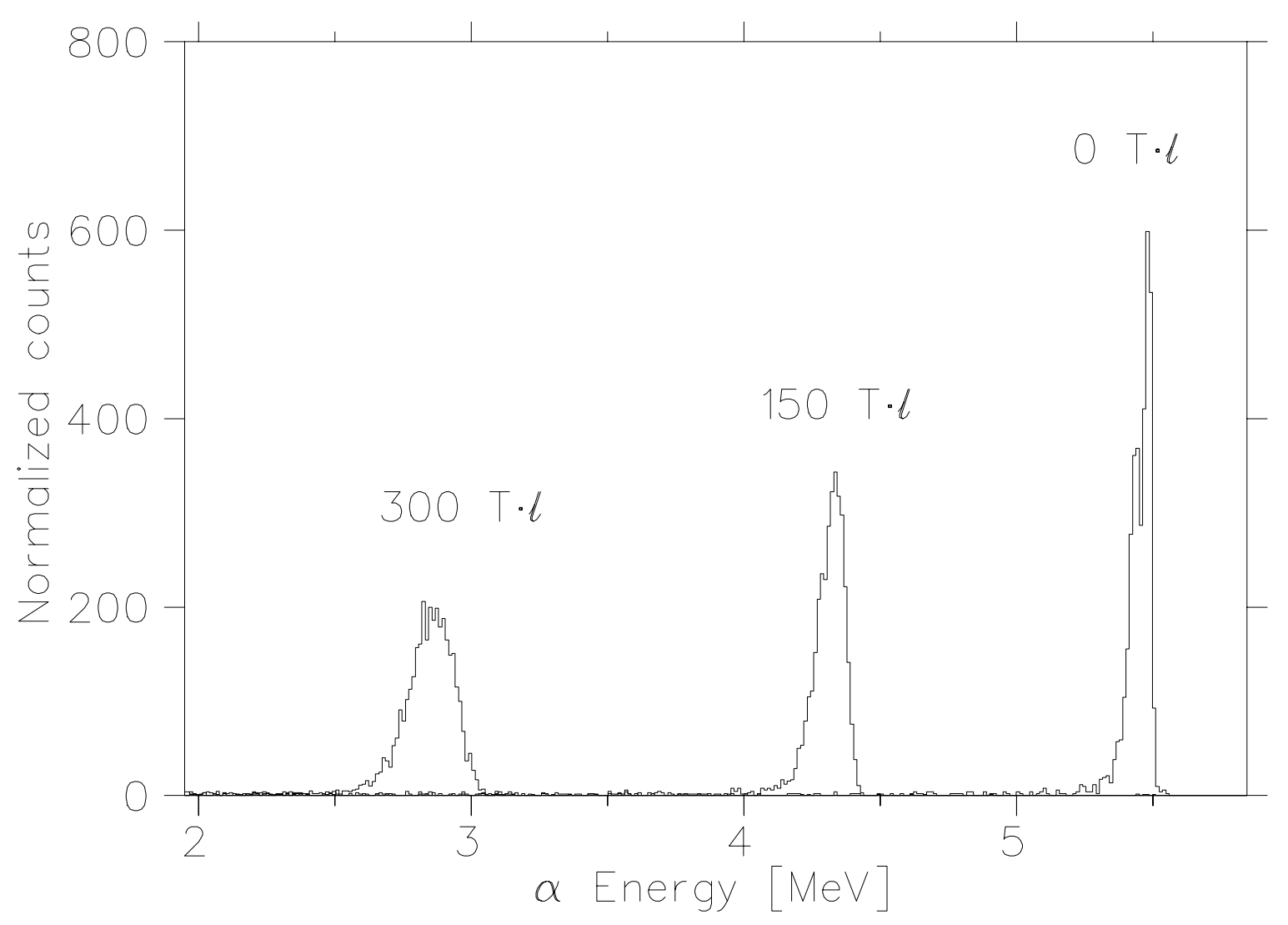

Fig. 3. Alpha particle energy spectra with different thicknesses of hydrogen film, where the peaks are normalized to the same number of counts. The numbers above each peak indicate the amount of hydrogen gas injected. 


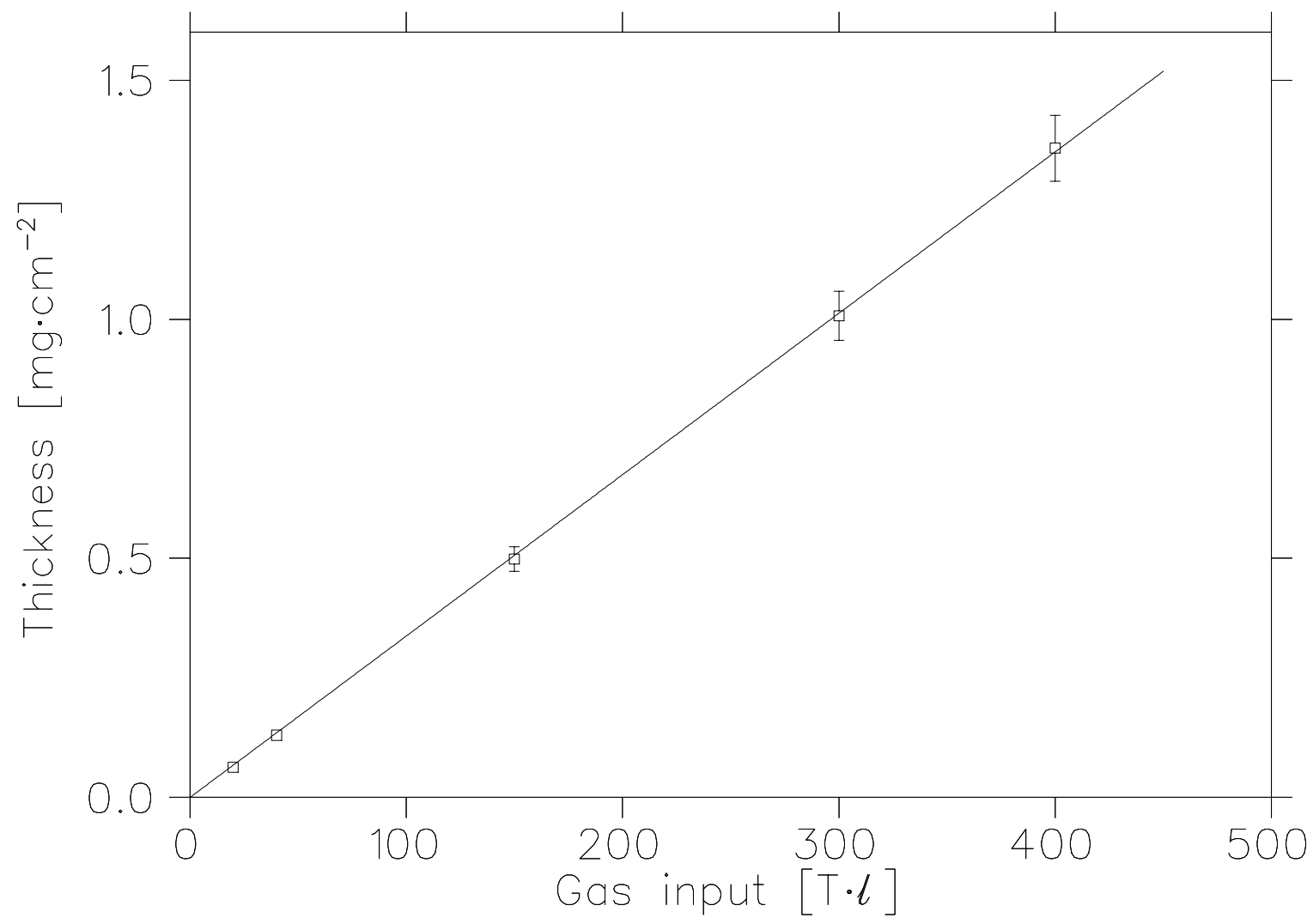

Fig. 4. Test of the linearity of deposition. The solid line represents a least-squares fit to the data points plotted with error bars. 


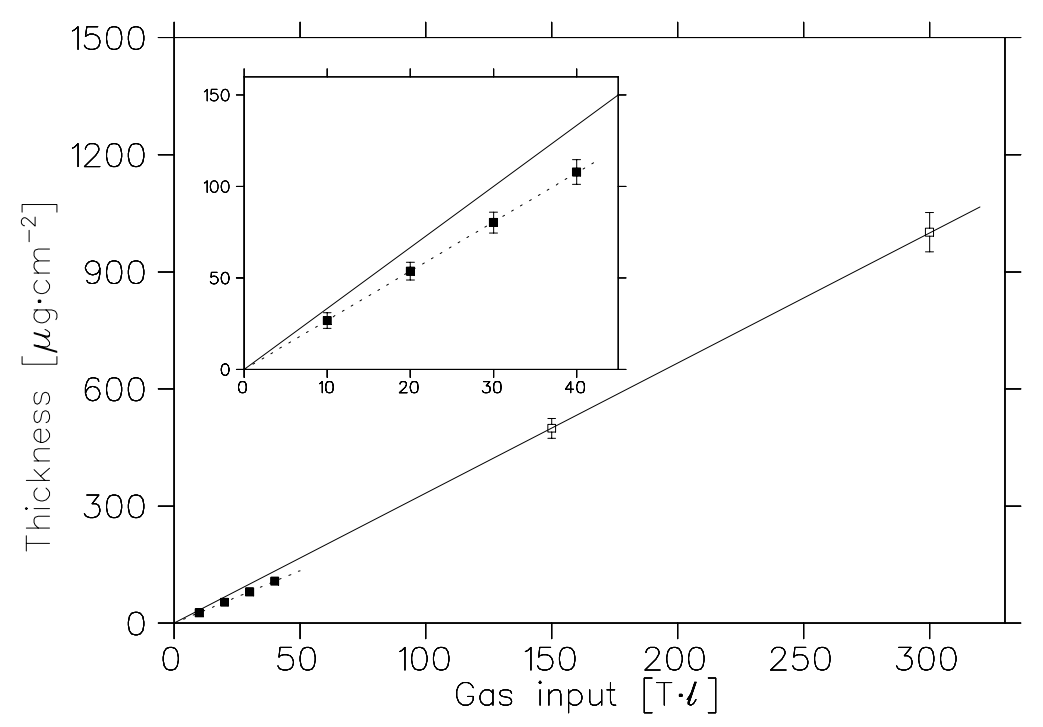

(a) System 2

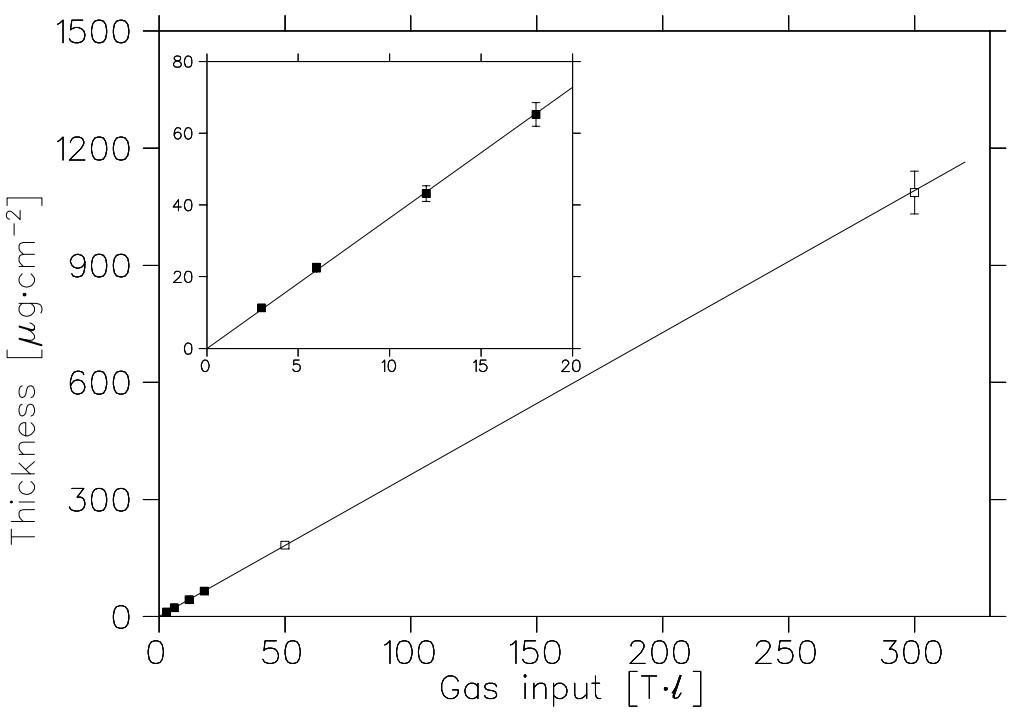

(b) System 3

Fig. 5. Sequential deposition of very thin films shown as filled squares is compared to the thick film deposition shown as open squares for different systems: (a) system 2 , where the difference in slopes between the two series of depositions indicates the gas loss effect, and (b) system 3, showing no evidence for such an effect. Inserted boxes in the figures illustrate details at small thicknesses. 


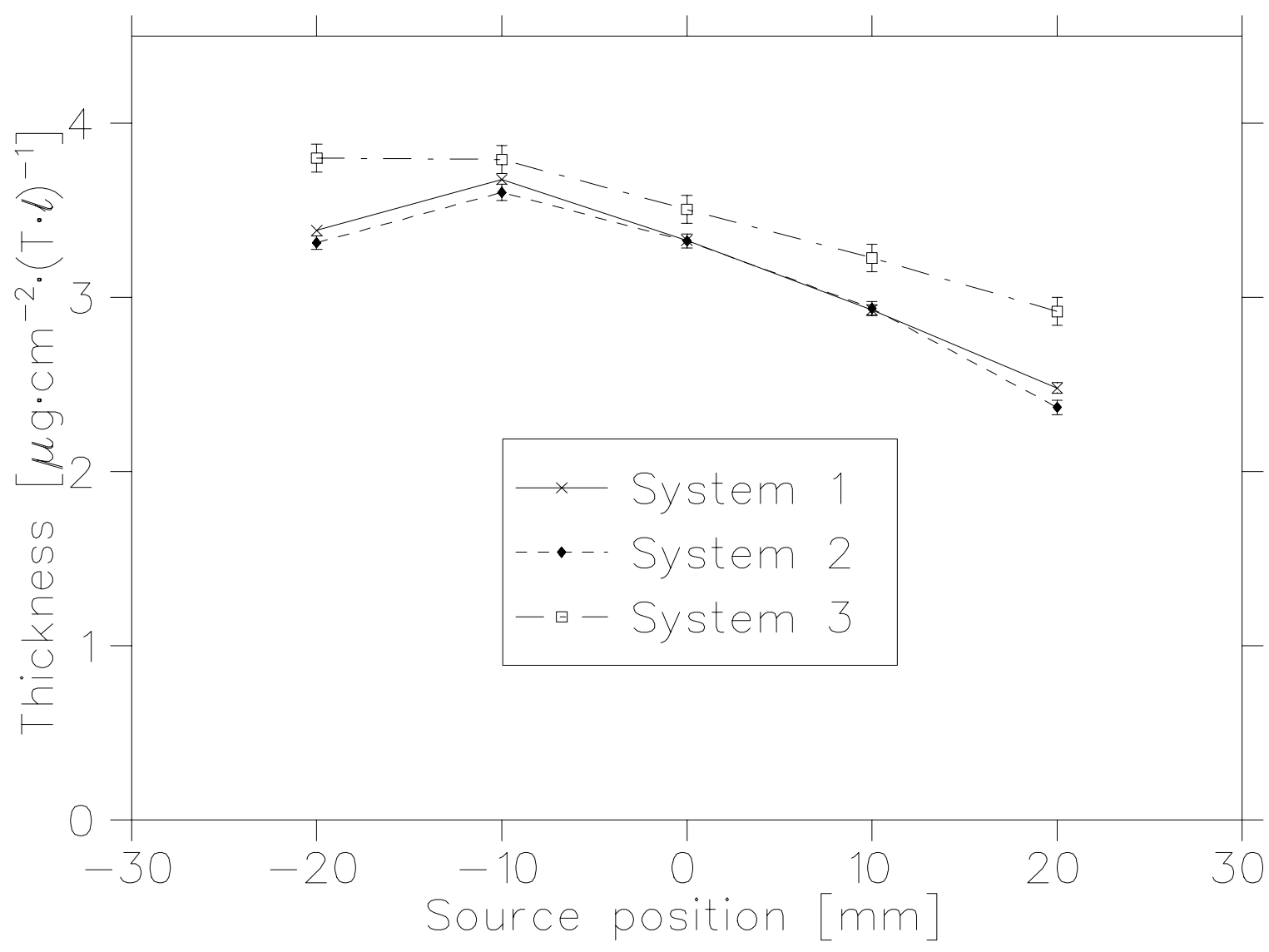

Fig. 6. Thickness profiles for different diffuser systems. Thickness per unit gas input is plotted against the source positions. Because these are relative measurements, the error bars do not include the uncertainty from the stopping power. 


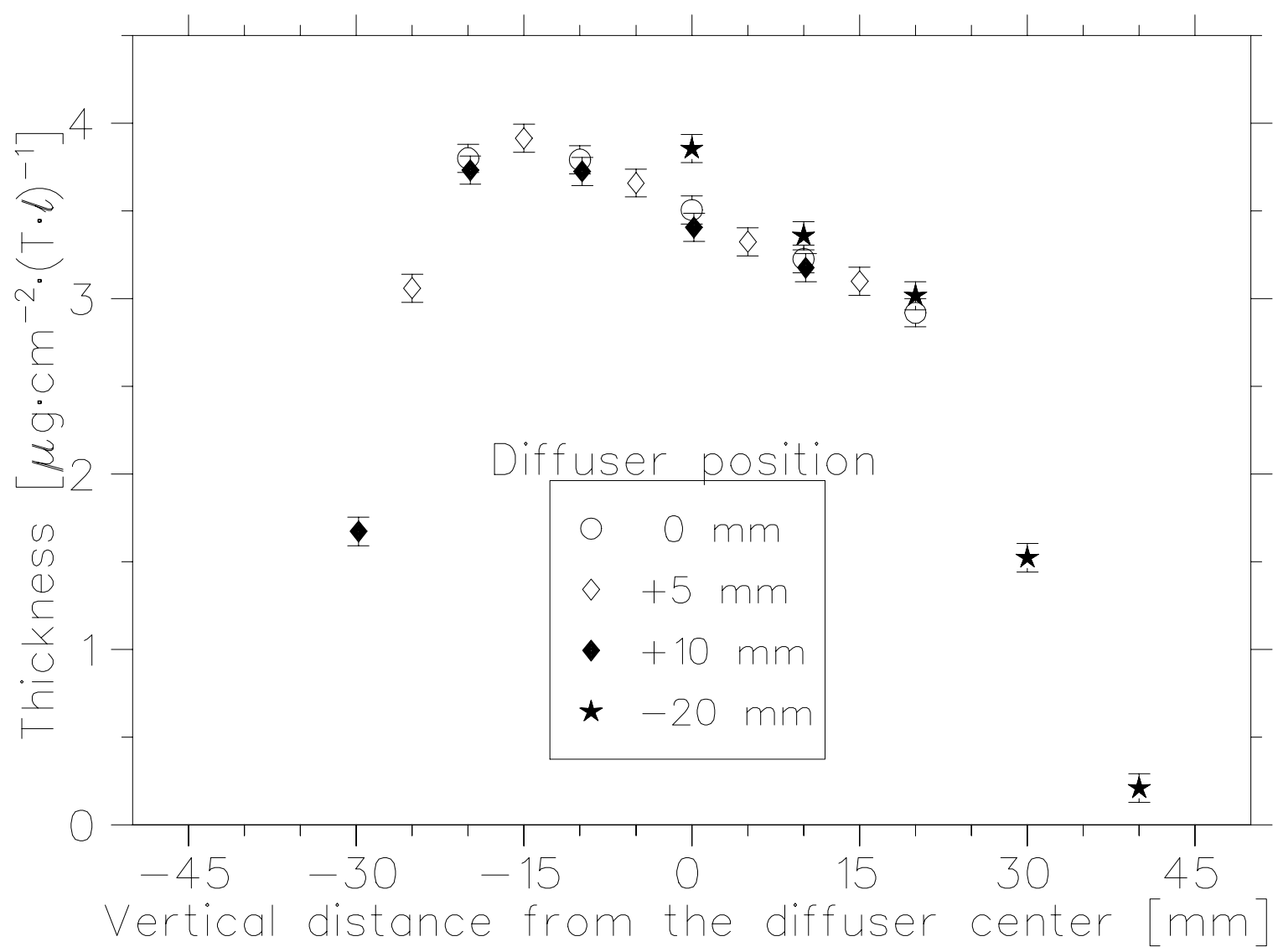

Fig. 7. Film thicknesses for system 3 are plotted against the vertical distance from the diffuser center (diffuser coordinates). The error bars do not include the uncertainty from the stopping power. See text for the detail. 


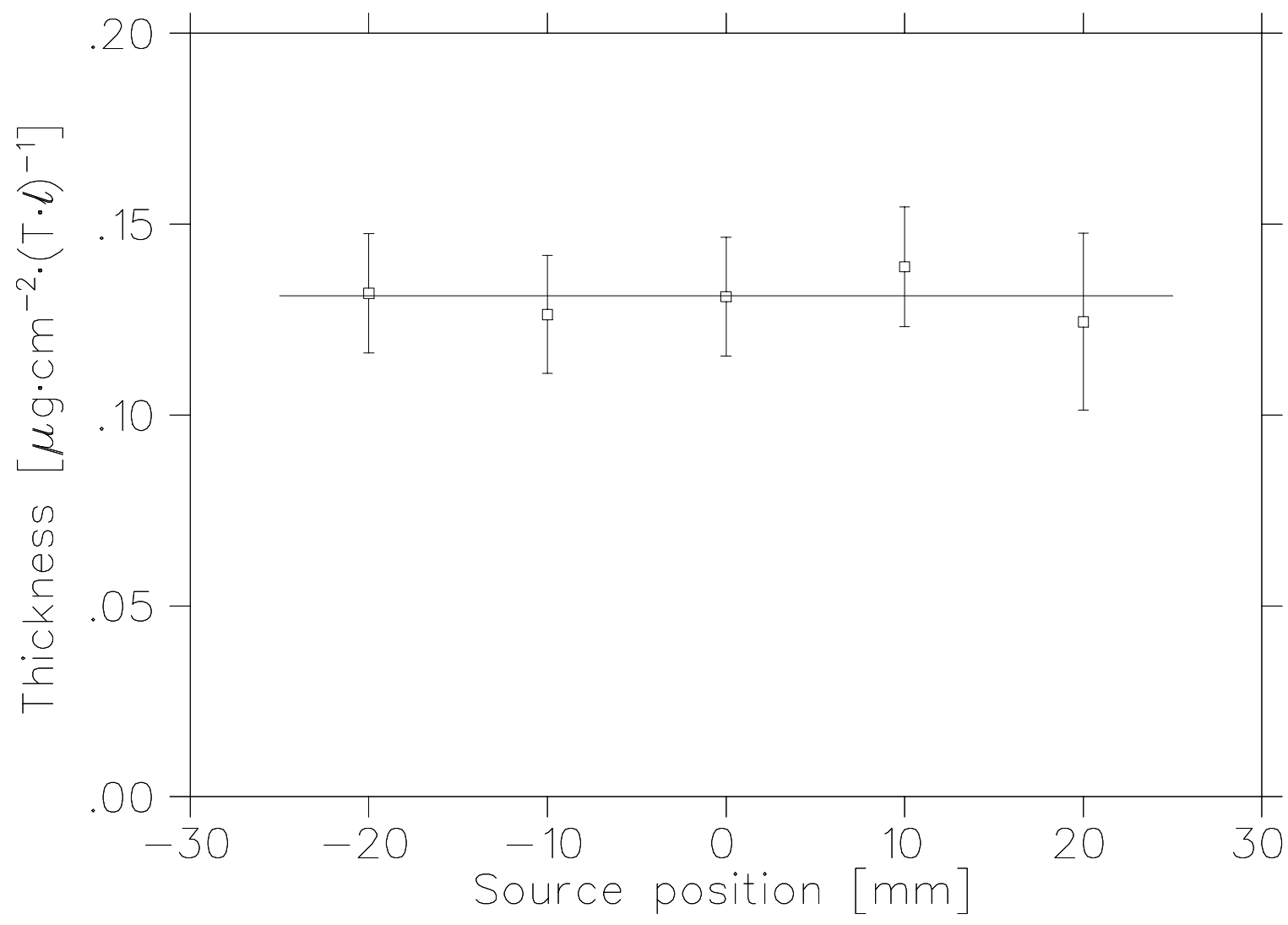

Fig. 8. Alternative deposition: Hydrogen gas was introduced to the entire cryostat with vacuum pump closed. The gas stuck uniformly to all of the cold surfaces. 


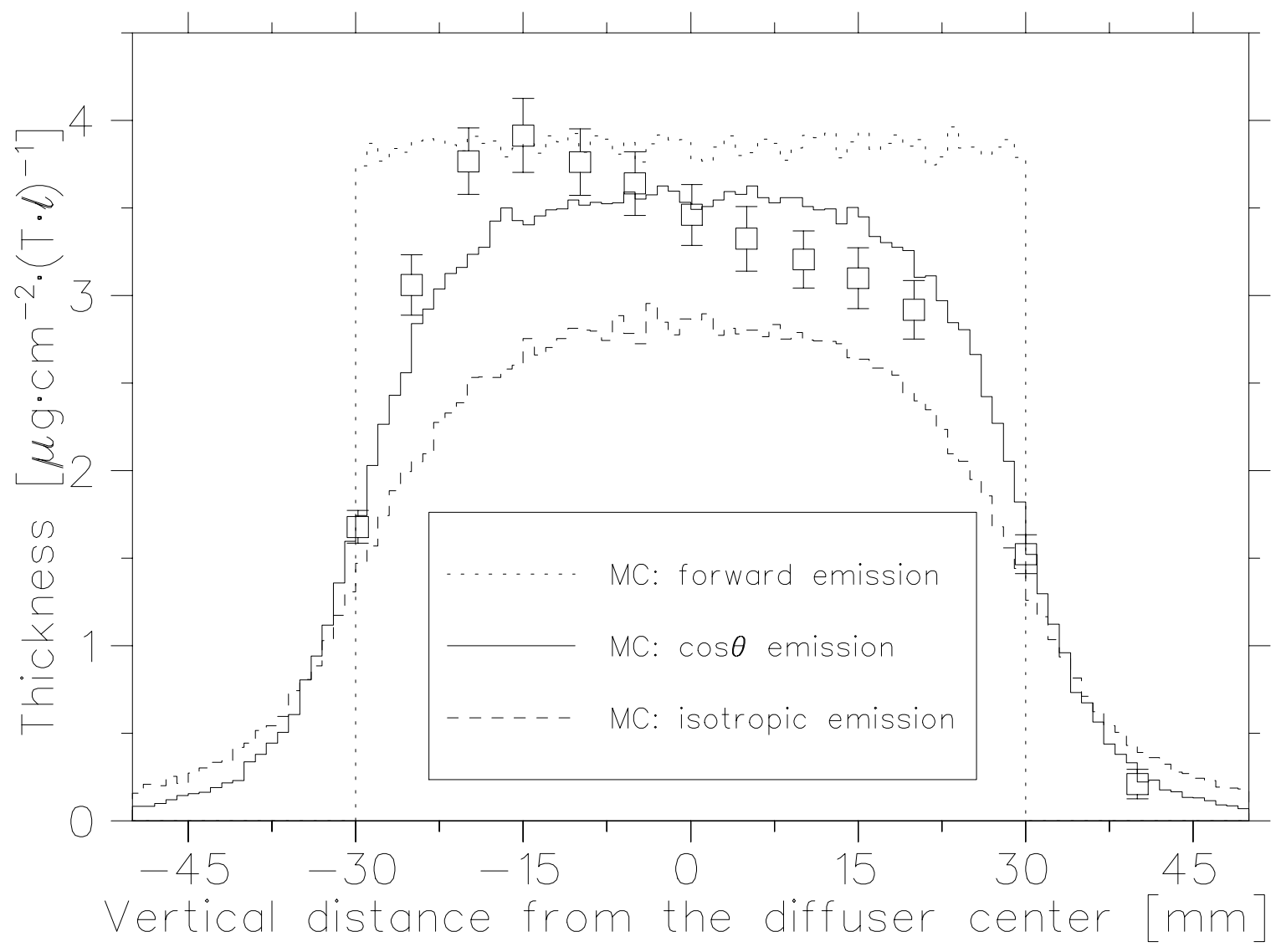

Fig. 9. The data, with error bars which include the uncertainty from the stopping power, denote the averaged thickness profile for system 3 plotted against the relative vertical distance from the diffuser center. The histograms are the the simulated thickness profiles from Monte Carlo calculations with different assumptions for the angular distribution of molecules emitted from the diffuser surface. 


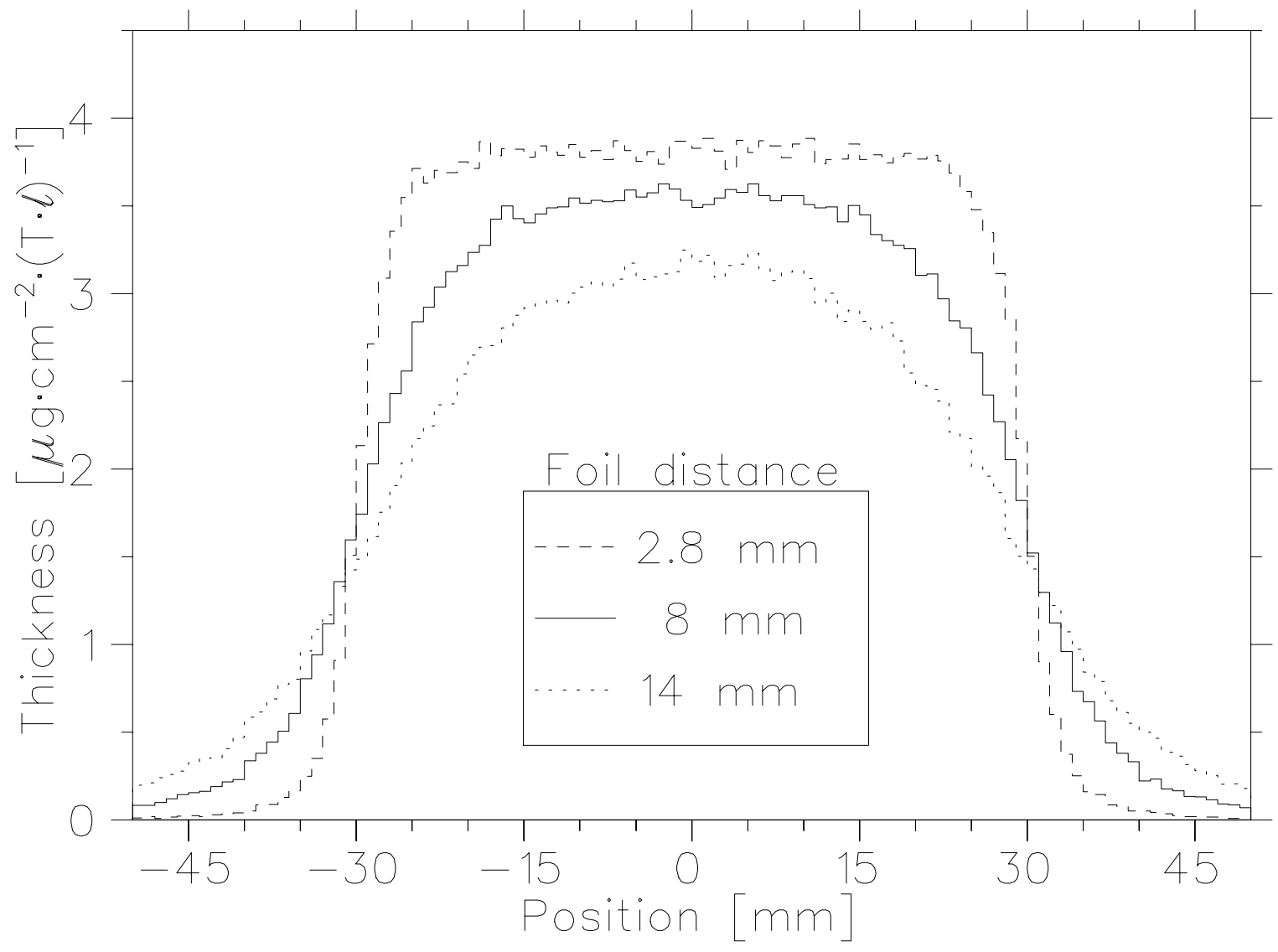

Fig. 10. Monte Carlo simulations comparing different distances between the diffuser surface and the cold foil surface. 